\title{
Epidemiological Characteristics of Strongyloidiasis in Inhabitants of Indigenous Communities in Borneo Island, Malaysia
}

\author{
Romano Nguil,", Noor Amira Abdul Halim¹, Yamuna Rajoo, ${ }^{1,2}$, Yvonne AL Lim¹, Stephen Ambu², Komalaveni Rajoo³, \\ Tey Siew Chang ${ }^{3}$, Lu Chan Woon ${ }^{3}$, Rohela Mahmud ${ }^{1}$ \\ 'Department of Parasitology, Faculty of Medicine, University of Malaya, Kuala Lumpur, Malaysia; ${ }^{2}$ International Medical University, No.126, Jalan \\ 19/155B, Bukit Jalil, 57000 Kuala Lumpur, Malaysia; ${ }^{3}$ Hospital Sarikei, Jalan Rentap 96100, Sarikei, Sarawak, Malaysia
}

\begin{abstract}
Epidemiological study on strongyloidiasis in humans is currently lacking in Malaysia. Thus, a cross-sectional study was carried out to determine the prevalence of Strongyloides stercoralis infection among the inhabitants of longhouse indigenous communities in Sarawak. A single stool and blood sample were collected from each participant and subjected to microscopy, serological and molecular techniques. Five species of intestinal parasites were identified by stool microscopy. None of the stool samples were positive for $S$. stercoralis. However, $11 \%$ of 236 serum samples were seropositive for strongyloidiasis. Further confirmation using molecular technique on stool samples of the seropositive individuals successfully amplified 5 samples, suggesting current active infections. The prevalence was significantly higher in adult males and tended to increase with age. S. stercoralis should no longer be neglected in any intestinal parasitic survey. Combination of more than 1 diagnostic technique is necessary to increase the likelihood of estimating the 'true' prevalence of $S$. stercoralis.
\end{abstract}

Key words: Strongyloides stercoralis, soil-transmitted helminth (STH), ELISA, PCR, Malaysia

The threadworm, Strongyloides stercoralis is a soil-transmitted helminth (STH) causing the disease strongyloidiasis. It is one of the most overlooked and neglected nematode infections, although an estimated 30-100 million people are infected worldwide [1-3]. It is endemic worldwide especially in the tropical and temperate zones as well as resource poor countries with inadequate environmental and sanitary conditions along with poor hygiene. Despite its high prevalence, the 'true' epidemiological data on the geographic distribution and global burden are largely lacking and scarce [3]. Its prevalence is often underestimated compared to other STHs species [1-3]. In Malaysia, there are only a few data on epidemiological studies of $S$. stercoralis are which available. Moreover, most of the previous studies were carried out mainly among rural communities in Peninsular Malaysia with prevalence rate ranging from $1.2 \%$ to $1.7 \%$ [4-6]. As for Thailand in particular, the epi-

\footnotetext{
- Received 20 March 2016, revised 11 July 2016, accepted 23 July 2016.

*Corresponding author (romano@um.edu.my)

(c) 2016, Korean Society for Parasitology and Tropical Medicine

This is an Open Access article distributed under the terms of the Creative Commons Attribution Non-Commercial License (http://creativecommons.org/licenses/by-nc/4.0) which permits unrestricted non-commercial use, distribution, and reproduction in any medium, provided the original work is properly cited.
}

demiology of $S$. stercoralis has been studied in some detail with prevalence ranging between $2.3 \%$ and $28.9 \%[7,8]$ and other countries, including Cambodia (10.3-21\%) [9,10], China (11.7\%) [11], and Japan (5-10\%) [12].

The difficult diagnosis and irregular excretion of larvae lead to an underreporting of its prevalence rates [13]. To date, there is no universally agreed gold standard for diagnosing $S$. stercoralis infection [3]. An important underlying reason is that one of the most widely used diagnostic techniques in parasitic epidemiological studies is simple microscopy examination which often fails to detect $S$. stercoralis, mainly due to its low sensitivity [14]. Concentration techniques [15] and larval cultivation [16] are more sensitive diagnostic tools which, however, are laborious, time consuming to perform, and not suitable to be used in large-scale epidemiological studies.

Alternatively, several serological approaches have also been developed to diagnose strongyloidiasis. In comparison to microscopy, serological method is sensitive but still suffers from high variables in specificity depending on the antigen and protocol used. Cross reactions with other STHs have also been reported with serological technique particularly in endemic areas where co-infection is common [7]. DNA-based methods 
such as PCR have been widely used, producing reliable results due to greater sensitivity and specificity and can unequivocally confirm current or past infections $[17,18]$.

Since there are limited studies on strongyloidiasis in Malaysia, we conducted an epidemiological study to determine the current prevalence of $S$. stercoralis infection among inhabitants of longhouse indigenous communities in Sarawak. To the best of our knowledge, this is the first study attempted to identify the occurrence of $S$. stercoralis among the community in East Malaysia.

A cross-sectional study was carried out in the indigenous community in Pakan town $\left(1.88333^{\circ} \mathrm{N}\right.$ latitude, $111.68333^{\circ} \mathrm{E}$ longitude), a small town located in the middle of Sarawak (Fig. 1). Three accessible longhouses were selected using a convenient sampling method. The sample size was calculated taking the prevalence anticipated and latest prevalence of $S$. stercolaris infections conducted in Malaysia [4]. The minimum sample size required was 222 participants with 95\% level of confidence and $5 \%$ bound on the error estimation.

The study protocol (i.e., MEC ID: 201401-0672) has been approved by the Ethics Committee of the University Malaya Medical Centre (UMMC), Malaysia. The aim and procedures of the study were explained to the villagers and their participation was voluntary. An informed consent sheet was signed by the head of the household or verbally for those who are illiterate followed by obtaining their thumb prints. Basic demographic information and clinical manifestation, such as nausea, diarrhea, and itchiness, associated with strongyloidiasis were obtained from a simple pre-tested questionnaire.
Stool samples were collected and preserved with $2.5 \%$ potassium dichromate in a wide-mouthed plastic stool container with a tight fitting lid pre-labeled with name and date of collection. An approximately $5 \mathrm{ml}$ of venous blood was collected from each respondent by trained medical assistants and nurses.

The stool samples were processed by standard direct smear and formalin-ether sedimentation techniques [19] followed by microscopic examinations. For serological test, a commercial ELISA kit (AccuDiag ${ }^{\mathrm{Tm}}$ Diagnostic Automation, Inc., Woodland Hills, California, USA) was used to detect IgG in the human serum. Stool samples of respondents whose serum samples were identified positive via ELISA were further analyzed to confirm the status of the infection using PCR. Briefly, a nested PCR which specifically amplified the internal transcribed spacer 1 (ITS1) region of the ribosomal DNA gene [17] was used for amplification on the extracted genomic DNA. Human glyceraldehyde-3-phosphate dehydrogenase (hGAPDH, accession no. XM005253678) was used as internal control. Data analysis was carried out using the SPSS software program for windows version 21 (SPSS, Chicago, Illinois, USA). Chi-square $\left(X^{2}\right)$ test on proportion was used to analyze the associations between binary and independent variables. The level of statistical significance was set at $P<0.05$ for each test.

A total of 236 respondents participated in this study with ages ranged between 1 to 91 years old (median age of 44 years). In this cohort, 111 (47\%) and 125 (53\%) were males and females, respectively. Five different intestinal parasitic pathogens were identified, including Ascaris lumbricoides, Trichuris trichiura, hookworms, Entamoeba spp., and Giardia lamblia.
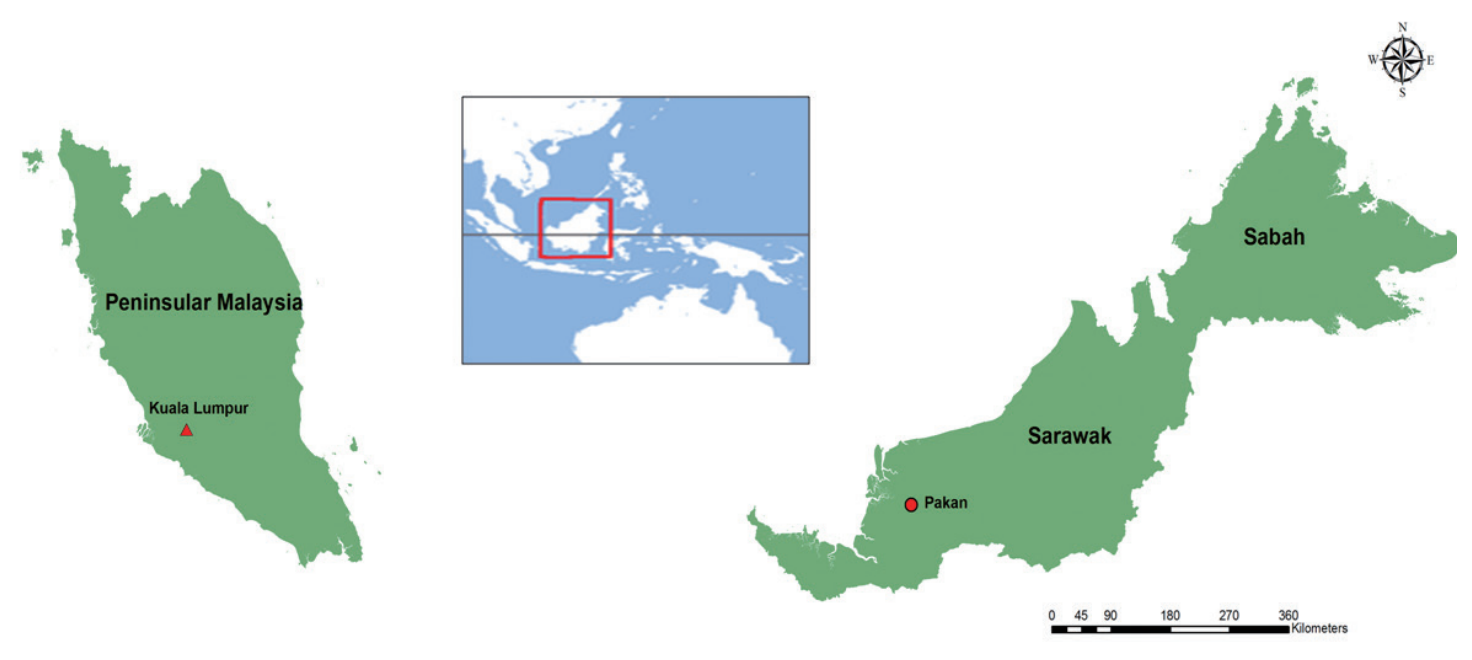

Fig. 1. Map of the surveyed area in Malaysia. 
Microcopy examinations, however, failed to identify S. stercoralis. However, of the 236 serum samples from the participants, 26 (11\%) were seropositive for strongyloidiasis (Table 1). Of these participants, 8 samples were also positive with other STH species. No other helminth species were identified in the remaining 18 positive samples. The seroprevalence of $S$. stercoralis was significantly higher in males than in females $(P=0.018)$. Similarly, the observed seroprevalence was significantly higher in adults than in children $(11.8 \%$ vs $4.0 \% ; P=0.041)$ and also increased with age $(P=0.039)$. No infections were found among toddlers and preschool children whereas $9.1 \%$ was recorded among school aged children and $11.1 \%$ among teenagers. The highest prevalence was reported among those aged 18 years and above (11.4\%) (Table 2).

Further confirmation using molecular method on the stool samples of seropositive individuals found that 5 (i.e., 4 males and 1 female) were positive by PCR, suggesting current active infections. No gastrointestinal or cutaneous symptoms were recorded from the individual positive by PCR as determined by questionnaire. In addition, none of these 5 samples were positive with other helminth species. Pools of 21 stool samples (i.e., 10 individual stool samples in each pool) from 210 sero-

Table 1. Prevalence of S. stercoralis infection detected by ELISA and PCR among indigenous longhouses communities $(n=236)^{a}$

\begin{tabular}{lccc}
\hline & \multicolumn{3}{c}{ S. stercoralis - PCR } \\
\cline { 2 - 4 } S. stercoralis - ELISA & Positive & Negative & Total \\
\hline Positive & 5 & 21 & 26 \\
Negative & 0 & 210 & 210 \\
Total & 5 & 231 & 236 \\
\hline
\end{tabular}

${ }^{a}$ None $(0 / 236)$ of the stool sample was positive for $S$. stercoralis larvae as examined by microscopy techniques (i.e., direct smear and formalinether sedimentation). negative individuals were also subjected to PCR; however, no DNA amplification was seen.

The prevalence of $S$. stercoralis varies significantly between continents and even within the same region, depending on the ecological and socioeconomic status $[3,13]$. Although $S$. stercoralis infection is known to be endemic in Southeast Asia, Thailand is the only country with an extensive amount of available data on strongyloidiasis [13]. In Malaysia, there is paucity of parasitological and epidemiological studies pertaining to $S$. stercoralis. In this study, a simple stool screening via direct smear and concentration techniques failed to identify any $S$. stercoralis infection. This finding is not surprising as previous studies on $S$. stercoralis conducted in Malaysia based on simple microscopy technique reported a very low prevalence of less than 2\% [4-6].

S. stercoralis infection is most often misdiagnosed and poorly characterized compared to other major STHs species, mainly due to the lack of suitable 'gold standard' diagnostic tools $[3,13]$. Basically, the standard diagnostic technique to diagnose strongyloidiasis is detection of the larvae in the stool. Nevertheless, in most uncomplicated cases, the worm load in the intestine of infected individual and the larvae output in the stool are both very low, making the diagnosis even more difficult [20]. The low sensitivity of microscopic techniques on a single stool collection for diagnosis of $S$. stercoralis is well known and may give false negative results $[3,11,14]$. Moreover, the larvae may be missed particularly in cases of light infections, which is common in asymptomatic individuals $[3,14]$. This may explain why the 'true' prevalence is often underestimated.

Previous studies have shown that examining multiple stool

Table 2. Prevalence of $S$. stercoralis as stratified by gender and age groups

\begin{tabular}{|c|c|c|c|c|c|}
\hline Characteristics & No. examined & No. positive & $\%$ & $\chi^{2}$ & $P$-value \\
\hline \multicolumn{6}{|l|}{ Gender } \\
\hline Male & 111 & 23 & 20.7 & & \\
\hline Female & 125 & 3 & 2.4 & 1.33 & 0.018 \\
\hline \multicolumn{6}{|l|}{ Age group (year) } \\
\hline Children (1-12) & 25 & 2 & 4.0 & & \\
\hline Teenager/Adults (13 and above) & 211 & 25 & 11.8 & 1.52 & 0.041 \\
\hline \multicolumn{6}{|l|}{ Age category (year) } \\
\hline 1-4 (toddlers) & 5 & 0 & 0 & & \\
\hline 5-6 (pre-school children) & 9 & 0 & 0 & & \\
\hline 7-12 (school aged children) & 11 & 1 & 9.1 & & \\
\hline 13-17 (teenagers) & 18 & 2 & 11.1 & & \\
\hline 18 and above (adults) & 193 & 23 & 11.9 & 3.60 & 0.039 \\
\hline
\end{tabular}


and cultivation techniques gave considerably higher sensitivity with an emphasis on S. stercoralis infection [11,14-16]. Such techniques and multiple stool examinations of at least 3 specimens collected over several days should be used in future studies to determine the 'true' prevalence of $S$. stercoralis infection. This is particularly important if the study design depends solely on stool microscopic examinations as the mean to identify the status of infection. Nonetheless, multiple sample collection was not being carried out in the present study mainly due to the lack of cooperation from the respondents. Similarly, such techniques were performed in the present study they requires fresh stool sample.

Given the previous limitations and poor performance of simple microscopy examination, we resorted to serological methods, which have high sensitivity and specificity. Among the various techniques, ELISA test has been shown to be superior with respect to practicality, safety, and greater sensitivity and specificity $[21,22]$. In this study, $11 \%$ serum specimens were ELISA positive. ELISA results show good sensitivity given that the study area is not endemic for filariasis as well as Schistosoma infection which is known to cross react with $S$. stercolaris [23]. However, potential cross reactions with other intestinal nematodes were of concern as the study area was also endemic for other intestinal nematodes $[24,25]$. This is not surprising given that both nematodes share similar modes of transmission. Other than that, positive ELISA results would not clearly prove the status of current active or past infections. Antibody presence alone cannot be used for diagnosis of active acute infections, since antibodies from prior exposure or past infection may circulate for a prolonged period of time after the infection has been cleared naturally or following anthelminthic treatment [24].

In recent years, PCR has been recognized and well established as a part of the highly sensitive and specific diagnostic tools to detect pathogens in clinical specimens, including $S$. stercoralis. In particular, when this nematode is difficult to culture and requires a long cultivation period, the diagnostic value of PCR is known to be significant. Moreover, PCR is able to diagnose strongyloidiasis in both asymptomatic and symptomatic individuals as shown in the present study. In this study, 5 stool samples from participants who were serologically positive were successfully amplified, while all other stool samples which were also subjected to PCR failed to produce any amplification. This finding may indirectly confirm the presence of current active infection of $S$. stercoralis, which coin- cide with other previous studies [4,17].

The clinical manifestations of strongyloidiasis vary greatly between individuals, depending on their immune status. The clinical features, including gastrointestinal and cutaneous symptoms are frequently associated with strongyloidiasis with more than a half of strongyloidiasis cases are asymptomatic $[1,2,9,13]$. Moreover, the clinical manifestations between strongyloidiasis and other infectious diseases particularly nematodes are not well described [13]. Thus, an in-depth assessment on the clinical symptoms of $S$. stercoralis infection is essential. In the present study, however, none of the positive individuals by PCR showed any subjective symptoms associated with S. stercolaris infection. Despite that these PCR-positive individuals were asymptomatic, preventive measures are warranted to decrease the impact of the disease, particularly among high risk groups as they may serve as the source of infective larvae. Thus, PCR is clinically significant especially among high risk groups, such as immunocompromised individuals with or without symptoms as it can cause hyperinfection and disseminated strongyloidiasis, which in many cases can be fatal.

In the present study, the observed prevalence of $S$. stercoralis was significantly higher in males than in females, which is in accordance with the previous study carried out among indigenous people in Peninsular Malaysia [4] as well as in other countries, including Cambodia $[9,10]$, Thailand $[7,8]$, China [11], and Taiwan [26]. This is most likely due to agricultural practices in the surveyed communities. In the present study, the majority of the respondents are involved with various farming activities, such as black pepper and hill rice cultivations. In contrast, most of the females are housewives.

The prevalence of $S$. stercoralis was also significantly higher in adults and also tended to increase with age, which coincided with the previous studies in Cambodia [9] and Thailand [7]. Similar age-pattern relationships have also been reported in China [11]. However, in Thailand, infections were also found among young children, aged between 1-6 years [8]. It is well known that infection and intensity of STHs infections are strongly age-dependent [27]. Only certain specific age groups are at a higher risk of acquiring infection although generally STHs can infect all age groups [28]. As for both hookworms and $S$. stercoralis, the intensity and frequency of infection show a steady rise with increasing age, peaking in adulthood.

The present study showed that $S$. stercoralis infection is an important medical problem that must not be neglected in any 
intestinal parasitic survey. Since all infected individuals are at risk for hyperinfection and a disseminated disease, the identification of people who may have contracted their infection many years earlier is of great clinical significance. Any individuals found to have an infection should be treated with anthelmintic drugs. All patients who are going to receive immunosuppressive drugs should be screened for strongyloidiasis before therapy. Like any other intestinal parasitic diseases, the best way to prevent $S$. stercoralis infection is to improve the environmental sanitation and personal hygiene, such as wear shoes when walking on soil and avoid contact with fecal matters. In addition, proper sewage disposal and fecal management along with anthelmintic drugs are keys for prevention.

In conclusion, further assessments in socioeconomic and ecological settings are also needed. An integration into global helminth control programs, such as health education and awareness campaign, application of more than 1 sensitive and specific techniques, compulsory screening of individuals from endemic areas especially among high risk groups, and development of novel diagnostic assays for rapid and accurate diagnosis of the disease, is warranted.

\section{ACKNOWLEDGMENTS}

We are grateful to the longhouses communities and local authorities of Sarikei Division. We deeply thank the Director of Sarikei Hospital for giving us the permission to use the laboratory. We also acknowledge the medical assistants, nurses, as well as laboratory technicians for their great field work. The study was supported by the University of Malaya grant (BKP 007-2014), Malaysia.

\section{CONFLICT OF INTEREST}

We have no conflict of interest related to this study.

\section{REFERENCES}

1. Bethony J, Brooker S, Albonico M, Geiger SM, Loukas A, Diemert D, Hotez PJ. Soil transmitted helminth infections: ascariasis, trichuriasis, and hookworm. Lancet 2006; 367: 1521-1532.

2. Hotez PJ, Molyneux DH, Fenwick A, Ottesen E, Sachs ES, Sachs JD. Incorporating a rapid-impact package for neglected tropical diseases with programs for HIV/AIDS, tuberculosis, and malaria. PLoS Med 2006; 3: e102.

3. Olsen A, van Lieshout L, Marti H, Polderman T, Polman K,
Steinmann P, Stothard R, Thybo S, Verweij JJ, Magnussen P. Strongyloidiasis-the most neglected of the neglected tropical diseases? Trans R Soc Trop Med Hyg 2009; 103: 967-972.

4. Ahmad AF, Hadip F, Ngui R, Lim YA, Mahmud R. Serological and molecular detection of Strongyloides stercoralis infection among Orang Asli community in Malaysia. Parasitol Res 2013; 112: 2811-2816.

5. Karim R, Rahmah N, Khairul Anuar A, Mehdi R, Abdullah B. Parasitic infections in the aboriginal community at Temenggor,Hulu Perak, Malaysia. Malay Nat J 1995; 48: 425-432.

6. Rahmah N, Ariff RH, Abdullah B, Shariman MS, Nazli MZ, Rizal MZ. Parasitic infections among aborigine children at Post Brooke, Kelantan, Malaysia. Med J Malaysia 1997; 52: 412-415.

7. Sithithaworn P, Srisawangwong T, Tesana S, Daenseekaew W, Sithithaworn J, Fujimaki Y, Ando K. Epidemiology of Strongyloides stercoralis in northeast Thailand: application of the agar plate culture technique compared with the enzyme-linked immunosorbent assay. Trans R Soc Trop Med Hyg 2003; 97: 398-402.

8. Nontasut P, Muennoo C, Sa-nguankiat S, Fongsri S, Vichit A. Prevalence of Strongyloides in Northern Thailand and treatment with ivermectin vs albendazole. Southeast Asian J Trop Med Public Health 2005; 36: 442-444.

9. Khieu V, Schar F, Marti H, Bless PJ, Char MC, Muth S, Odermatt P. Prevalence and risk factors of Strongyloides stercoralis in Takeo Province, Cambodia. Parasit Vectors 2014; 7: 221.

10. Sayasone S, Vonghajack Y, Vanmany M, Rasphone O, Tesana S, Utzinger J, Akkhavong K, Odermatt P. Diversity of human intestinal helminthiasis in Lao PDR. Trans R Soc Trop Med Hyg 2009; 103: 247-254.

11. Steinmann P, Zhou XN, Du ZW, Jiang JY, Wang LB, Wang XZ, Li $\mathrm{LH}$, Marti H, Utzinger J. Occurrence of Strongyloides stercoralis in Yunnan Province, China, and comparison of diagnostic methods. PLoS Negl Trop Dis 2007; 1: e75.

12. Ohta N, Waikagul J. Disease burden and epidemiology of soiltransmitted helminthiases and schistosomiasis in Asia: the Japanese perspective. Trends Parasitol 2007; 23: 30-35.

13. Schar F, Trostdorf U, Giardina F, Khieu V, Muth S, Marti H, Vounatsou P, Odermatt P. Strongyloides stercoralis: Global Distribution and Risk Factors. PLoS Negl Trop Dis 2013; 7: e2288.

14. Ericsson CD, Steffen R, Siddiqui AA, Berk SL. Diagnosis of Strongyloides stercoralis Infection. Clin Infect Dis 2001; 33: 1040-1047.

15. Garcia LS. Diagnostic Medical Parasitology. Washington DC, USA. ASM Press. 2001, pp. 791.

16. Koga K, Kasuya S, Khamboonruang C, Sukhavat K, Ieda M, Takatsuka N, Kita K, Ohtomo H. A modified agar plate method for detection of Strongyloides stercoralis. Am J Trop Med Hyg 1991; 45: 518-521.

17. Nilforoushan MR, Mirhendi H, Rezaie S, Rezaian M, Meamar AR, Kia EB. A DNA-Based identification of Strongyloides stercoralis isolates from Iran. Iran J Pub Health 2007; 36: 16-20.

18. Verweij JJ, Canales M, Polman K, Ziem J, Brienen EA, Polderman AM, van Lieshout L. Molecular diagnosis of Strongyloides stercoralis in faecal samples using real-time PCR. Trans R Soc Trop Med 
Hyg 2009; 103: 342-346.

19. Chessbrough M. Parasitological Test: District Laboratory Practice in Tropical Countries, Part 1. Cambridge, UK. Cambridge University Press. 1998.

20. Rayan HZ, Soliman RH, Galal NM. Detection of Strongyloides stercoralis in fecal samples using conventional parasitological techniques and Real-Time PCR: a comparative study. Parasitol United J 2012; 5: 27-34.

21. Liu LX, Weller PF. Strongyloidiasis and other intestinal nematode infections. Infect Dis Clin North Am 1993; 7: 655-682.

22. Paula FM, Costa-Cruz JM. Epidemiological aspects of strongyloidiasis in Brazil. Parasitology 2011; 138: 1331-1340.

23. Norsyahida A, Riazi M, Sadjjadi SM, Muhammad Hafiznur Y, Low HC, Zeehaida M, Noordin R. Laboratory detection of strongyloidiasis: IgG-, IgG4 - and IgE-ELISAs and cross-reactivity with lymphatic filariasis. Parasite Immunol 2013; 35: 174-179.

24. Yori PP, Kosek M, Gilman RH, Cordova J, Bern C, Chavez CB,
Olortegui MP, Montalvan C, Sanchez GM, Worthen B, Worthen J, Leung F, Ore CV. Seroepidemiology of strongyloidiasis in the Peruvian Amazon. Am J Trop Med Hyg 2006; 74: 97-102.

25. Hall A, Conway DJ, Anwar KS, Rahman ML. Strongyloides stercoralis in an urban slum community in Bangladesh: factors independently associated with infection. Trans R Soc Trop Med Hyg 1994; 88: 527-530.

26. Wu TC, Lei WY, Chen MC, Hu CT. Strongyloides stercoralis infection: a health issue regarding indigenous people in Taiwan. Trans R Soc Trop Med Hyg 2012; 106: 468-472.

27. Traub RJ, Robertson ID, Irwin P, Mencke N, Thompson RC. The role of dogs in transmission of gastrointestinal parasites in a remote tea-growing community in northeastern India. Am J Trop Med Hyg 2002; 67: 539-545.

28. Anderson RM, May RM, Anderson B. Infectious diseases of humans: dynamics and control. Oxford, UK. Oxford University Press. 1992. 